\title{
Diagnosis of gastric adenocarcinoma using a scoring system: Combined assay of serological markers of Helicobacter pylori infection, pepsinogen $I$ and gastrin
}

\author{
Jaw-Town Lin, Wen-Chung Lee, Ming-Shiang Wu, Jin-Town Wang, Teh-Hong Wang, and Chien-Jen Chen \\ Department of Internal Medicine, College of Medicine and Institute of Public Health, College of Public Health, National Taiwan University, \\ Taipei, Taiwan
}

\begin{abstract}
This study was carried out to develop a scoring system for the diagnosis of gastric adenocarcinoma (GAC). A total of 686 subjects, 150 patients with GAC, 182 with gastric ulcer, 127 with duodenal ulcer, and 227 subjects with negative findings, were enrolled. Analysis of the likelihood ratio (LR) showed that patients with advanced age, ulcer in the stomach, low serum levels of pepsinogen I (PGI), low PGI $\times$ gastrin values, and low $\mathrm{PGI} /$ gastrin ratio were likely to have GAC. Of these indicators, the serum PGI level had the greatest weight, with a LR of 7.59 for the group with a level $<30 \mathrm{ng} / \mathrm{ml}$. A scoring system combining serum PGI level, Helicobacter pylori seropositivity, and gastric ulcer status was derived, using a logistic regression model. This scoring system was found to be better than any one-parameter criterion for diagnosing GAC after evaluation by the area under the receiver operating characteristic curve $(0.84$; $95 \%$ confidence interval, $0.81-0.88$ ) or by specificityfixed sensitivity (sensitivity 0.82 at specificity 0.72 , sensitivity 0.87 at specificity 0.66 , sensitivity 0.96 at specificity 0.44 ). This scoring system may be potentially useful as a new model for the noninvasive diagnosis of GAC in the future.
\end{abstract}

Key words: pepsinogen I, $H$. pylori, gastric adenocarcinoma, scoring system

Offprint requests to: J.-T. Lin, Department of Internal Medicine, National Taiwan University Hospital, No. 7, Chung-Shan S. Rd., Sec. 1, Taipei, Taiwan 10017.

(Received for publication on Apr. 12, 1994; accepted on July 22, 1994)

\section{Introduction}

Gastric adenocarcinoma (GAC) is one of the most common cancers worldwide. ${ }^{1}$ The diagnosis of GAC is based mainly on endoscopy combined with histologic examination. The invasiveness of endoscopy generally makes it non-feasible for the screening of asymptomatic subjects. Except in Japan, where mass screening programs are successfully performed, the majority of GAC are found at an advanced stage and the patients have a poor prognosis. ${ }^{2}$ Various tumor markers have been evaluated for the early diagnosis of GAC; however, their low sensitivity and specificity precludes their clinical use for the early detection of this disease. ${ }^{3-5}$

Previous reports have shown that shown that serum pepsinogen I (PGI) levels are low in patients with atrophic gastritis and $\mathrm{GAC},{ }^{6-9}$ but that serum gastrin levels are elevated in these patients. ${ }^{10,11} \mathrm{~A}$ combination of these two markers may significantly improve the diagnostic ability for detecting severe atrophic gastritis and, probably, GAC. ${ }^{12}$ Recently, it has been reported that Helicobacter pylori infection is also associated with GAC. ${ }^{13,14}$ However, serum levels of gastrin and PGI were reported to be affected by $H$. pylori infection. ${ }^{15,16}$ The complex interaction among these factors indicates that using any oneparameter criterion for the diagnosis of GAC would usually be inadequate, and a combined assay of $H$. pylori seropositivity and serum levels of PGI and gastrin may be needed.

In this study, a scoring system using these three serologic markers, as well as other clinical variables, was developed. The predictive power and diagnostic value of the proposed system are potentially useful as a new model for the noninvasive diagnosis of GAC in the future. 


\section{Materials and methods}

\section{Subjects}

Between January 1992 and June 1993, a total of 686 subjects who underwent endoscopic examination in the National Taiwan University Hospital were enrolled in the present study. They were free from major cardiopulmonary, hepatobiliary, and renal diseases. None of them had received gastric surgery, or were receiving ongoing treatment with antibiotics, acid reducing agents, or nonsteroidal antiinflammatory drugs. One hundred and fifty of the 686 subjects were diagnosed histologically as having GAC. The nonGAC controls (536 subjects) consisted of 227 negative controls with normal gastric mucosa or minimal gastritis (determined by endoscopy after the exclusion of macroscopic abnormalities, including intestinal metaplasia, atrophic gastritis, erosions, ulcers, polyps, and tumors in the stomach and duodenal bulb), 182 patients with endoscopically and histologically confirmed benign gastric ulcer, and 127 patients with endoscopically verified duodenal ulcer (Table 1). Two hundred and forty patients with peptic ulcer had endoscopic evidence of antral gastritis.

\section{Laboratory methods}

Fasting blood samples, obtained from all subjects on the morning of their first endoscopy, were stored at $-70^{\circ} \mathrm{C}$ until examination. IgG antibodies against H. pylori were tested using an enzyme-linked immunosorbent assay (ELISA) (HEL-p test; AMRAD, Victoria, Australia). The antigen was an inactivated native antigen of $H$. pylori. The manufacturer's state that this assay has a sensitivity of $96 \%$ and a specificity of $93 \%$. The serum level of PGI was determined with a commercial radioimmunoassay kit (Sorin Biomedica, Saluggia, Italy), was the serum level of gastrin (Incstar Corp., Stillwater, Minn.). Serum PGI and gastrin levels were expressed as means $\pm \mathrm{SD}$.

\section{Statistical analysis}

Evaluation of single diagnostic test (one-parameter criterion). Various laboratory and clinical characteristics of the study subjects were evaluated with regard to their diagnostic value in GAC. As some test results were repeated on continuous scales, the likelihood ratios (LRs), calculated by dividing the proportion of GAC patients with a given result by the proportion of non-GAC subjects with the same result, were employed in this study instead. ${ }^{17}$ Ninetyfive percent confidence intervals (CI) for the LRs were also determined. ${ }^{18}$ The posterior odds (and hence the posterior probability) of having GAC for a subject with a result " $x$ " were calculated by the following formula: ${ }^{17}$

Posterior odds of GAC at $x=$ (prior odds) $\times($ LR at $x)$

Construction of a scoring system. A binary logistic model was used to examine how GAC occurrence depends on laboratory results and any covariates of interest. A forward stepwise algorithm was employed to select the significant predictors. The variables evaluated were: sex (male/female); age (categorized into five 10-year levels); ulcer in the stomach (yes/no); H. pylori seropositivity (yes/no); serum PGI; serum

Table 1. Demographic data, $H$. pylori seropositivity, and serum levels of pepsinogen I and gastrin in gastric adenocarcinoma $(G A C)$ cases and non-GAC subjects

\begin{tabular}{lcccc}
\hline & & \multicolumn{3}{c}{ Non-GAC subjects $(n=536)$} \\
\cline { 4 - 5 } & GAC cases & & \multicolumn{3}{c}{$(n=150)$} & $\mathrm{GU}(n=182)$ & $\mathrm{DU}(n=127)$ & $\mathrm{NC}(n=227)$ \\
\hline Age in years & $59.2 \pm 14.7$ & $59.5 \pm 12.3$ & $48.1 \pm 12.3^{*}$ & $46.6 \pm 13.5^{*}$ \\
$\quad$ (range) & $(24-87)$ & $(26-88)$ & $(18-83)$ & $(18-79)$ \\
$\begin{array}{l}\text { Sex ratio } \\
\text { Male/Female }\end{array}$ & $102 / 48$ & $131 / 51$ & $82 / 45$ & $87 / 140^{* *}$ \\
H.pylori positivity & $91 / 150$ & $148 / 182$ & $108 / 127$ & $129 / 227$ \\
$\begin{array}{c}\text { (\%) } \\
\text { Serum PGI in ng/ml }\end{array}$ & $(60.7)$ & $(81.3)$ & $(85.0)$ & $(56.8)$ \\
$\quad$ (range) & $(4.6 \pm 36.9$ & $101.9 \pm 53.9^{*}$ & $94.1 \pm 36.7^{*}$ & $66.0 \pm 24.0$ \\
Serum GS in pg/ml & $62.2 \pm 29.0$ & $(9.2-271)$ & $(18.5-254)$ & $(19.2-140)$ \\
(range) & $(12.6-184)$ & $(21-233)$ & $(33.7-161)$ & $(18.1-245)$ \\
\hline
\end{tabular}

${ }^{*} P<0.005$ vs GAC by Student's $t$-test; ${ }^{* *} P<0.005$ vs GAC by chi-square test with Yate's correction

$G A C$, Gastric adenocarcinoma; $G U$, gastric ulcer; $D U$, duodenal ulcer; $N C$, negative control;

$P G I$, pepsinogen I; $G S$, gastrin; $H$. pylori positivity, no. of subjects with positive IgG antibody

to $H$. pylori/no. subjects examined

All data are expressed as means $\pm \mathrm{SD}$ 
gastrin; the product PGI $\times$ gastrin; and the ratio $\mathrm{PGI} /$ gastrin. Each of the last four factors were categorized into four levels. The significance level for entering the model was set at a type 1 error of 0.05 . The analysis was performed by the SAS/LOGISTIC program. ${ }^{19}$ After the final model was selected, the "intercept" estimated from the model was subtracted by logarithm (prior odds of GAC), that is, $\log (150 / 536)=-1.27 .^{20}$ All the parameters in the model were then exponentiated, rescaled, and rounded to serve as clinically useful scores. The scores corresponding to each level of the diagnostic criteria for a subject can be multiplied together to produce a composite score. This composite score also represents the model-based LR of being a GAC case for that particular subject. ${ }^{20}$ Based on this scoring formula, any subject could be classified into a "case" or a "non-case" category if his/her composite score (or LR) was greater or less than one. A desired level of sensitivity (or specificity) can also be achieved by varying the cut-off point for the composite score.

Evaluation of the scoring system. To evaluate the predictive power of the scoring system, GAC cases and non-GAC subjects in this study were separately grouped into five categories according to their composite scores derived from the model. The expected number of subjects in each category was calculated and compared to the, observed number. The goodnessof-fit test of Hosmer and Lemeshow was applied. ${ }^{21}$

To compare the diagnostic values of the scoring system with any one-parameter criterion, areas under the receiver operating characteristic (ROC) curves (AUC) were used as the performance indices. ${ }^{22}$ The AUCs were calculated by the trapezoid rule. ${ }^{22} \mathrm{~A}$ nonparametric statistical test was used for the comparison. ${ }^{23}$ With regard to discrete diagnostic tests, we fixed the specificity at the level of the discrete test and compared the estimated sensitivity of the scoring system with the sensitivity of the discrete test. ${ }^{24}$ The sensitivity of the scoring system at a particular level of specificity was estimated by linear intrapolation if required.

\section{Results}

\section{Clinical and laboratory characteristics}

As shown in Table 1, patients with GAC were significantly older than the patients with duodenal ulcer $(P<0.005)$ and the negative controls $(P<0.005)$. Female predominance was noted only in the negative controls (male/female, 87/140, $P<0.005$ vs GAC). No significant difference in $H$. pylori seropositivity was observed between GAC cases and non-GAC subjects. The serum PGI levels were significantly higher in patients with gastric ulcer $(101.9 \pm 53.9$ $\mathrm{ng} / \mathrm{ml})$ and duodenal ulcer $(94.1 \pm 36.7 \mathrm{ng} / \mathrm{ml})$ than in those with GAC $(61.9 \pm 36.9 \mathrm{ng} / \mathrm{ml})(P<0.005)$. Serum gastrin levels in GAC cases were not significantly different from those in patients with gastric or duodenal ulcer, or the negative controls.

\section{Diagnosis of gastric adenocarcinoma (GAC) with a single parameter}

The LRs of diagnosing GAC by clinical characteristics and/or laboratory results are shown in Table 2. Subjects with advanced age, ulcer in the stomach, low levels of serum PGI, low PGI $\times$ gastrin values, and low PGI $/$ gastrin ratios were likely to have GAC. Of these variables, serum PGI level had the greatest weight, with LRs ranging from 7.59 to 0.56 .

Table 2. Likelihood ratios $(L R)$ wth $95 \%$ confidence intervals $(C I)$ of subjects being gastric adenocarcinoma cases by clinical characteristics and laboratory results

\begin{tabular}{|c|c|c|c|c|}
\hline & $\begin{array}{l}\text { GAC } \\
\text { cases }\end{array}$ & $\begin{array}{c}\text { Non-GAC } \\
\text { subjects }\end{array}$ & LR & $95 \% \mathrm{CI}$ \\
\hline \multicolumn{5}{|l|}{ Sex } \\
\hline Female & 48 & 236 & 0.73 & $0.56-0.94$ \\
\hline Male & 102 & 300 & 1.21 & $1.06-1.39$ \\
\hline \multicolumn{5}{|l|}{ Age (years) } \\
\hline$\leq 40$ & 24 & 122 & 0.70 & $0.47-1.05$ \\
\hline $41-50$ & 15 & 126 & 0.43 & $0.26-0.70$ \\
\hline $51-60$ & 30 & 127 & 0.84 & $0.59-1.20$ \\
\hline $61-70$ & 48 & 124 & 1.38 & $1.05-1.83$ \\
\hline$>70$ & 33 & 37 & 3.19 & $2.07-4.91$ \\
\hline \multicolumn{5}{|c|}{ Ulcer in the stomach } \\
\hline No & 25 & 354 & 0.25 & $0.18-0.36$ \\
\hline Yes & 125 & 182 & 2.45 & $2.14-2.82$ \\
\hline \multicolumn{5}{|l|}{ H. pylori } \\
\hline Negative & 59 & 151 & 1.40 & $1.10-1.78$ \\
\hline Positive & 91 & 385 & 1.84 & $0.73-0.97$ \\
\hline \multicolumn{5}{|l|}{ PGI (ng/ml) } \\
\hline$\leq 30$ & 34 & 16 & 7.59 & $4.31-13.37$ \\
\hline $31-50$ & 29 & 77 & 1.35 & $0.91-1.98$ \\
\hline $51-80$ & 48 & 195 & 0.88 & $0.68-1.14$ \\
\hline$>80$ & 39 & 248 & 0.56 & $0.42-0.75$ \\
\hline \multicolumn{5}{|c|}{ Gastrin $(\mathrm{pg} / \mathrm{ml})$} \\
\hline$\leq 40$ & 32 & 81 & 1.41 & $0.98-2.04$ \\
\hline $41-60$ & 54 & 207 & 0.93 & $0.73-1.18$ \\
\hline $61-80$ & 31 & 129 & 0.86 & $0.61-1.22$ \\
\hline$>80$ & 33 & 119 & 0.99 & $0.70-1.39$ \\
\hline \multicolumn{5}{|c|}{ PGI $\times$ gastrin value } \\
\hline$\leq 2000$ & 45 & 48 & 3.35 & $2.33-4.82$ \\
\hline $2001-4000$ & 50 & 181 & 0.99 & $0.76-1.27$ \\
\hline $4001-6000$ & 25 & 151 & 0.59 & $0.40-0.87$ \\
\hline$>6000$ & 30 & 156 & 0.69 & $0.49-0.97$ \\
\hline \multicolumn{5}{|c|}{ PGI/gastrin ratio } \\
\hline$\leq 0.6$ & 43 & 53 & 2.90 & $2.02-4.15$ \\
\hline $0.61-1.20$ & 48 & 188 & 0.91 & $0.70-1.18$ \\
\hline $1.21-1.80$ & 36 & 138 & 0.93 & $0.68-1.28$ \\
\hline$>1.80$ & 23 & 157 & 0.52 & $0.35-0.78$ \\
\hline
\end{tabular}

PGI, Pepsinogen I 
Diagnosis of gastric adenocarcinoma (GAC) with the scoring system

The scoring system derived from the logistic regression model is shown in Table 3. The background score was 7.2 for every subject. Presence of ulcer in the stomach, absence of $H$. pylori infection, and lower serum levels of PGI were associated with increased scores.

Table 4 shows the predictive power of this scoring system: The expected number of subjects in each category as predicted by the model was in close agreement with the observed number (goodness-of-fit test $P=0.1841$ ). The performance of the scoring system as compared to various one-parameter criteria is shown in Table 5. The AUC of the scoring system was $0.84(95 \% \mathrm{CI} 0.81-0.88)$, with a sensitivity of 0.82 at specificity 0.72 , sensitivity 0.87 at specificity 0.66 , and sensitivity 0.96 at specificity 0.44 . The scoring system was better than any one-parameter criterion, as evaluated by AUC or by specificity-fixed sensitivity. This superiority reached a significant level, except

Table 3. Scoring system ${ }^{\text {a }}$ derived from the logistic regression model

\begin{tabular}{lc}
\hline Terms & Score \\
\hline Background score & 7.2 \\
Ulcer in the stomach & \\
$\quad$ No & 0.1 \\
Yes & 1.4 \\
Pepsinogen I (ng/ml) & \\
$\quad \leq 30$ & 2.0 \\
$31-50$ & 0.4 \\
$51-80$ & 0.2 \\
$>80$ & 0.1 \\
H. pylori & \\
Negative & 1.8 \\
Positive & 1.0
\end{tabular}

${ }^{a}$ Scores corresponding to each level of the diagnostic results for a subject can be multiplied together to produce a composite score, which is also the likelihood ratio for being a GAC case for the subject under evaluation

Table 4. Observed $(O b s)$ vs expected (Exp) number of GAC cases and non-GAC subjects by composite score category $^{\mathrm{a}}$

\begin{tabular}{lccr}
$\begin{array}{l}\text { Composite } \\
\text { score category }\end{array}$ & $\begin{array}{c}\text { GAC cases } \\
\text { Obs (Exp) }\end{array}$ & $\begin{array}{c}\text { non-GAC subjects } \\
\text { Obs (Exp) }\end{array}$ & Total \\
\hline$\leq 0.25$ & $6(7.1)$ & $234(232.9)$ & 240 \\
$0.26-0.50$ & $11(6.2)$ & $77(81.8)$ & 88 \\
$0.51-2.00$ & $41(42.6)$ & $146(144.4)$ & 187 \\
$2.01-4.00$ & $43(39.9)$ & $57(60.1)$ & 100 \\
$\geq 4.00$ & $49(50.6)$ & $22(20.4)$ & 71 \\
Total & $150(146.3)$ & $536(539.7)$ & 686 \\
\hline
\end{tabular}

Table 5. Comparison of performance of the scoring system and various one-parameter criteria

\begin{tabular}{lcc}
\hline $\begin{array}{l}\text { Diagnostic test or } \\
\text { clinical characterisitcs }\end{array}$ & AUC $(95 \% \text { CI })^{\mathrm{a}}$ & $\begin{array}{c}\text { Sensitivity } \\
\text { Specificity }\end{array}$ \\
\hline Scoring system & $0.84(0.81-0.88)$ & $0.82 / 0.72$ \\
& & $0.87 / 0.66$ \\
& & $0.96 / 0.44$ \\
One-parameter criterion & $0.66(0.61-0.71)^{*}$ & - \\
PGI & $0.53(0.47-0.58)^{*}$ & - \\
Gastrin & $0.65(0.60-0.71)^{*}$ & - \\
PGI $\times$ gastrin value & $0.62(0.57-0.67)^{*}$ & - \\
PGI/gastrin ratio & $0.65(0.60-0.71)^{*}$ & - \\
Age & - & $0.39 / 0.72^{*}$ \\
H. pylori seropositivity & - & $0.83 / 0.66$ \\
Ulcer in stomach & - & $0.68 / 0.44^{*}$ \\
Sex & & \\
\hline
\end{tabular}

${ }^{*} P<0.0001$, compared with scoring system

$A U C$, Areas under receiver operating characteristics (ROC) curves; $95 \% C I, 95 \%$ confidence interval; $P G I$, pepsinogen I

when compared to the variable of the presence of ulcer in the stomach.

\section{Discussion}

In this study, the demographic data of GAC patients were in line with other series. ${ }^{2}$ Although the seroprevalence $(60.7 \%)$ of $H$. pylori infection in GAC cases was not significantly different from that in the negative controls $(56.8 \%)$, the seropositivity of patients with gastric ulcer and duodenal ulcer was higher than that in the GAC cases. This result is in accordance with the hypothesis that $H$. pylori infection may be associated with peptic ulcer, but is not sufficient for gastric carcinogenesis. ${ }^{25}$ Findings similar to ours have also been reported. ${ }^{26-29}$ The lower serum PGI levels in GAC observed in this study are also consistent with previous reports and thus suggest that PGI may be a potential serologic marker for GAC. ${ }^{8,9}$ Nevertheless, there is a considerable overlapping in PGI between GAC and benign conditions. Various other factors, such as the gastric inflammation induced by $H$. pylori infection, may also increase serum PGI. ${ }^{16}$ Therefore, the diagnosis of GAC using this single parameter may not be satisfactory.

Based on our LR analysis, advanced age, ulcer in the stomach, low serum PGI level, low PGI $\times$ gastrin values, and low $\mathrm{PGI} /$ gastrin ratio were associated with an increased likelihood of having GAC. Serum PGI level less than $30 \mathrm{ng} / \mathrm{ml}$ had the highest LR (7.59) and had the greatest weight. This cut-off-point $(30 \mathrm{ng} / \mathrm{ml})$ was also mentioned in a previous report on atrophic gastritis. ${ }^{30}$ However, in contrast to the study of Farinati et al. ${ }^{12}$ neither the PGI $\times$ gastrin value nor the PGI/gastrin ratio was better than PGI alone in 
predicting the risk for GAC in this study. Gastrin alone was also not of diagnostic value in our study.

In our scoring system, the presence of ulcer in the stomach increased the composite score. This finding should not, however, be interpreted as reflecting gastric cancers arising from pre-existing benign ulcer. It may simply indicate that, in our study, the gastric malignancy was generally of the ulcerative type, rather than of the fungating type. The role played by $H$. pylori in our scoring system also deserves close scrutiny. In our system, negative $\operatorname{IgG}$ antibody to $H$. pylori increased the composite score. This apparently paradoxical result may be due to a complex interaction between $H$. pylori seropositivity and age. ${ }^{26}$ In general, although the prevalence of $H$. pylori infection has been found to increase with age, it tends to decline after an advanced age is reached. ${ }^{16,26}$ The predominance of aged patients and the relatively low $H$. pylori seropositivity in GAC in this study may explain why $H$. pylori seronegativity increased the composite score and, thus, the GAC risk. Further analysis, using tree-structured classification, ${ }^{31}$ may clarify the role played by $H$. pylori in gastric carcinogenesis and thus improve our scoring system.

The markers that predict GAC in this scoring system are clinically simple to use. It is convenient to draw blood and measure the serologic markers of $H$. pylori and PGI. To recognize the presence of gastric ulcer, a non-invasive examination such as doublecontrast upper gastrointestinal radiology may be employed. Based on the above methods, any subject can easily be classified as a "GAC suspect" or a "nonGAC subject" if their composite scores are greater or less than one. As this composite score system also represents the model-based LR of having GAC for a particular subject, in may be useful for the targetting of high-risk patients for additional investigation. Furthermore, it may serve as a supplemental means to distinguish a benign from a malignant gastric ulcer when radiology alone is controversial.

PGI was a slighty more favorable marker than others in our study. Nevertheless, the PGI/PGII ratio, rather than PGI alone, has recently been demonstrated to have greater weight for estimating the severity and/or extent of atrophic gastritis, and to be more predictive of gastric cancer. ${ }^{9,30,32}$ Therefore, further investigations to increase the predictive value of our model by combining other serum markers, such as PGII, are mandatory in the future.

This study was a preliminary attempt to analyze the clinical usefulness of various serum markers and demographic characteristics for the diagnosis of GAC from a new perspective. The scoring system we have proposed awaits further evaluation in different clinical settings and in different patient populations.
Acknowledgment. This study was supported by grants from the Taipei Institute of Pathology, Department of Health, Executive Yuan (DOH-84-TD-010), Taiwan, and the National. Science Council (NSC-83-0412-B002-151), Taipei, Taiwan.

\section{References}

1. Parkin DM, Laara E, Muir CS. Estimates of the worldwide frequency of sixteen major cancers in 1980. Int J Cancer 1988; 41:184-197.

2. Dupont J, Lee J, Burton $G$, et al. Adenocarcinoma of the stomach: Review of 1497 cases. Cancer 1978;41:941-947.

3. Ellis DJ, Speirs C, Kingston RD, et al. Carcinoembryonic antigen levels in advanced gastric carcinoma. Cancer 1978;42: $623-625$.

4. Ritts RE, Del Villano BC, Go VLW, et al. Initial clinical evaluation of an immunoradiometric assay for CA19-9 using the NCI serum bank. Int J Cancer 1984;33:339-345.

5. Wobbes $T$, Thomas $C$, Segers $M$, Nagengast $F$. Evaluation of seven tumor markers (CA50, CA19-9, CA19-9 Truquant, CA72-4, CA195, carcinoembryonic antigen, and tissue polypeptide antigen) in the pretreatment sera of patients with gastric carcinoma. Cancer 1992;69:2036-2041.

6. Samloff IM, Varis K, Ihamäki T, et al. Relationships among serum pepsinogen I, serum pepsinogen II, and gastric mucosal histology: A study in relatives of patients with pernicious anemia. Gastroenterology 1982;83:204-209.

7. Varis K, Samloff IM, Ihamäki T, Siurala M. An appraisal of tests for severe atrophic gastritis in relatives of patients with pernicious anemia. Dig Dis Sci 1979;24:187-191.

8. Nomura A, Stemmerman GN, Samloff IM. Serum pepsinogen I as a predictor of stomach cancer. Ann Intern Med 1980;93: $537-540$.

9. Stemmermann GN, Samloff IM, Nomura A, Heilbrun LK. Serum pepsinogen I and II and stomach cancer. Clin Chim Acta 1987;163:191-198.

10. Sipponen P, Valle J, Varis K, et al. Fasting levels of serum gastrin in different functional and morphological states of the antrofundal mucosa: An analysis of 860 subjects. Scand J Gastroenterol 1990;25:513-519.

11. McGuigan JE, Trudeau WKL. Serum and tissue gastrin concentrations in patients with carcinoma of the stomach. Gastroenterology 1973;64:22-25.

12. Farinati F, Mario F, Plebani M, et al. Pepsinogen A/pepsinogen C or pepsinogen A multiplied by gastrin in the diagnosis of gastric cancer. Ital J Gastroenterol 1991;223:194-196.

13. Parsonnet J, Friedman GD, Vandersteen DP, et al. Helicobacter pylori infeciton and the risk of gastric carcinoma. N Engl J Med 1991;325:1127-1131.

14. Nomura A, Stemmerman GN, Chyou P, et al. Helicobacter pylori infection and gastric carcinoma among Japanese Americans in Hawaii. N Engl J Med 1991;325:1132-1136.

15. Smith JTL, Pounder RE, Nwokole CU, et al. Inappropriate hypergastrinemia in asymptomatic healthy subjects with Helicobacter pylori. Gut 1990;31:522-525.

16. Asaka M, Kimura $\mathrm{T}$, Kudo $\mathrm{M}$, et al. Relationship of Helicobacter pylori to serum pepsinogens in an asymptomatic Japanese population, Gastroenterology 1992;102:760-766.

17. Simel DL, Samsa GP, Matchar DB. Likelihood ratios for continuous test results: Making the clinician's job easier or harder? J Clin Epidemiol 1993;46:85-93.

18. Simel DL, Samsa GP, Matchar DB. Likelihood ratios with confidence: Sample size estimation for diagnostic test studies. J Clin Epidemiol 1994;44:763-770.

19. SAS Technical Reoprt P-229, SAS/STAT software: LOGISTIC Procedures (release 6.07). Cary, NC, U.S.A., 1992. 
20. Irwig L. Modelling result-specific likelihood ratios. J Clin Epidemiol 1992;45:1135-1140.

21. Hosmer DW, Lemeshow S. Goodness-of-fit tests for the multiple logistic regression model. Commun Statist Theor Meth 1980;A9(10):1043-1069.

22. Hanley JA, McNeil BJ. The meaning and use of the area under a receiver operating characteristic (ROC) curve. Radiology 1982;143:29-36.

23. DeLong ER, DeLong DM, Clarke-Pearson DL. Comparing the areas under two or more correlated receiver operating characteristic curves: A nonparametric approach. Biometrics 1988;44: 837-845.

24. Beam CA, Wieand HS. A statistical method for the comparison of a discrete diagnostic test with several continuous diagnostic tests. Biometrics 1991;47:907-919.

25. Correa $\mathrm{P}$. Is gastric carcinoma an infectious disease? N Engl J Med 1991;325:1170-1171.

26. Kuipers EJ, Gracia-Casanova M, Peña AS, et al. Helicobacter pylori serology in patients with gastric carcinoma. Scand J Gastroenterol 1993;28:433-437.

27. Sierra R, Muñoz N, Peña AS, et al. Antibodies to Helicobacter pylori and pepsinogen levels in children from Costa Rica:
Comparison of two areas with different risks for stomach cancer. Cancer Epidemiol Biomark Prev 1992;1:449-454.

28. Lin JT, Wang JT, Wang TH, et al. Helicobacter pylori infection in a randomly selected population, healthy volunteers, and patients with gastric ulcer and gastric adenocarcinoma: A seroprevalence study in Taiwan. Scand J Gastroenterol 1993;28: 1067-1072.

29. Lin JT, Wang JT, Wang TH, Wu MS, Chen CJ. Helicobacter pylori infection in early and advanced gastric adenocarcinoma: A seroprevalence study in 143 .Taiwanese patients. Hepatogastroenterology 1993;40:596-599.

30. Kekki M, Samloff IM, Varis K, Ihamäki T. Serum pepsinogen I and serum gastrin in the screening of severe atrophic corpus gastritis. Scand J Gastroenterol 1991;26[Suppl 186]:109116.

31. Cook EF, Goldman L. Empiric comparison of multivariate analytic techniques: Advantages and disadvantages of recursive partitioning analysis. J Chron Dis 1984;37:721-731.

32. Miki K, Ichinose M, Ishikawa KB, et al. Clinical application of serum pepsinogen I and II levels for mass screening to detect gastric cancer. Jpn I Cancer Res 1993;84:1086-1090. 